\title{
CAUCHY PROBLEM AND REDUCTION OF A SYSTEM OF PARTIAL DIFFERENTIAL EQUATIONS
}

\author{
J. STEFANOVSKI, K. TRENC̆EVSKI AND V. COVACHEV
}

\begin{abstract}
The Cauchy problem for systems of homogeneous and also nonlinear partial differential equations is considered. If the compatibility conditions are satisfied, the solution is represented as functional series. The algorithm for reduction of a system of partial differential equations with linear homogeneous algebraic constraints is considered. It is proved that the compatibility conditions for the reduced system are identically satisfied.
\end{abstract}

\section{Introduction}

In the paper vector notations are used, where the vectors and matrices are in bold. For this notation we assume that the differential (row) operator $\frac{\partial}{\partial \mathbf{x}}$ denotes $\left[\frac{\partial}{\partial x_{1}}, \ldots, \frac{\partial}{\partial x_{n}}\right]$. For example, if $\varphi$ is a scalar function, then

$$
\frac{\partial \varphi}{\partial \mathbf{x}}=\left[\frac{\partial \varphi}{\partial x_{1}}, \ldots, \frac{\partial \varphi}{\partial x_{n}}\right]
$$

and for any vector function $\mathfrak{h}\left(x_{1}, \ldots, x_{n}\right)=\left[\begin{array}{c}h_{1} \\ \vdots \\ h_{m}\end{array}\right]$ we use the notation

$$
\frac{\partial \mathbf{h}}{\partial \mathbf{x}}=\left[\begin{array}{cccc}
\partial h_{1} / \partial x_{1} & \partial h_{1} / \partial x_{2} & \cdots & \partial h_{1} / \partial x_{n} \\
\partial h_{2} / \partial x_{1} & \partial h_{2} / \partial x_{2} & \cdots & \partial h_{2} / \partial x_{n} \\
\vdots & \vdots & \cdots & \vdots \\
\partial h_{m} / \partial x_{1} & \partial h_{m} / \partial x_{2} & \cdots & \partial h_{m} / \partial x_{n}
\end{array}\right]
$$

This paper is based on the papers $[5,6,7,8]$ and represents a further generalization of them. So we will give a brief overview of these papers.

Received February 4, 1999; revised May 5, 1999.

1991 Mathematics Subject Classification. 35F05, 35F20

Key words and phrases. Cauchy problem, system of partial differential equations, algebraic constraints.

This work is supported by the Ministry of Sciences of $R$. Macedonia within the project "Differential Geometry and Applications" under the Contract 08-0007/4, and by the Ministry of Education and Sciences of $R$. Bulgaria within the project "Asymptotic and Geometric Methods in Spectral Theory and Scattering Theory" under Grant MM-706/97. 
In [6] a Charpit system of partial differential equations (PDEs)

$$
\frac{\partial \mathrm{y}}{\partial \mathbf{x}} \cdot \mathbf{h}_{i}(\mathbf{x}, \mathbf{y})+\mathbf{g}_{i}(\mathbf{x}, \mathbf{y})=0, \quad i=1, \ldots, p
$$

is considered together with the algebraic constraints

$$
\mathfrak{f}(\mathrm{x}, \mathrm{y})=\mathfrak{f}(\mathrm{z})=\mathbf{0}
$$

where the unknown vector function $\mathrm{y}$ is $m$-dimensional and depends on the $n$-dimensional variable $\mathbf{x}$, while $\mathbf{h}_{i}, \mathbf{g}_{i}$, $\mathbf{f}$ are given vector functions. Then it is expanded up to the equivalent system with respect to the unknown function $F(\mathbf{x}, \mathbf{y})=F(\mathbf{z})$ :

$$
\frac{\partial F}{\partial \mathbf{z}} \cdot \mathbf{w}_{\mathbf{i}}(\mathbf{z})=\mathbf{0}, \quad i=1, \ldots, q, \quad \mathbf{z}=\left[\begin{array}{l}
\mathbf{x} \\
\mathbf{y}
\end{array}\right]
$$

where $\mathbf{w}_{i}(\mathbf{z})=\left[\begin{array}{l}\boldsymbol{h}_{i} \\ -\mathrm{g}_{i}\end{array}\right]$, for some $q \geq p$, so that (1.3) identically satisfies the compatiblity conditions, and it is proven [6] that if the solution of (1.1) satisfies the algebraic equations (1.2), then it also satisfies the algebraic equations

$$
\frac{\partial \mathbf{f}}{\partial \mathbf{z}} \cdot \mathbf{w}_{i}(\mathbf{z})=\mathbf{0}, \quad i=1, \ldots, q .
$$

We generate other algebraic equations, besides the equations (1.2). Let us put $\mathbf{f}_{0}(\mathbb{z})=$ $f_{0}(x, y)=f(x, y)=f(z)=0$ and let us assume inductively that we have obtained the vector equation $\tilde{f}_{j}(z)=0$. Let us sort the components of $\mathfrak{f}_{j}$ into $\overline{\mathfrak{f}}_{j}$ and $\tilde{\mathfrak{f}}_{j}$ so that the rows of the matrix $\partial \overline{\mathfrak{f}}_{j} / \partial \mathrm{z}$ be linearly independent and the rows of the matrix $\partial \tilde{\mathfrak{f}}_{j} / \partial \mathrm{z}$ be linearly dependent on the rows of the matrix $\partial \overline{\mathfrak{f}}_{j} / \partial \mathrm{z}$. While generating the vector functions $\overline{\mathfrak{f}}_{j}$ let us assume that the components of $\mathfrak{f}_{j}$ are ordered so the the first components of $\overline{\mathbb{f}}_{j}$ are the components of $\overline{\mathbb{f}}_{j-1}$. Applying the previous assertion to the equations $\overline{\mathfrak{f}}_{j}(\mathbb{z})=0$, new equations can be obtained. Let us put

$$
\mathfrak{f}_{j+1}=\left[\begin{array}{c}
\overline{\mathfrak{f}}_{j} \\
\left(\partial \overline{\mathfrak{f}}_{j} / \partial \mathbf{z}\right) \cdot \mathbf{w}_{1} \\
\cdots \\
\left(\partial \overline{\mathfrak{f}}_{j} / \partial \mathbf{z}\right) \cdot \mathbf{w}_{q}
\end{array}\right]=\mathbf{0}
$$

This completes the description of the algorithm.

A necessary condition for existence of a solution of (1.1) and (1.2) is the existence of points $\mathbb{z}_{j}$ such that

$$
\overline{\mathfrak{f}}_{j}\left(\mathbf{z}_{j}\right)=0, \quad \tilde{\mathbb{f}}_{j}\left(\mathbf{z}_{j}\right)=0, \quad j=0,1, \ldots
$$

As a result of the algorithm we obtain that the number of components of the vector function $\overline{\mathbb{f}}_{j}$ permanently increases and its first components are the components of the previous function $\overline{\mathfrak{f}}_{j-1}$. There exists a minimal positive integer $k$ such that $\overline{\mathfrak{f}}_{k+1}=\overline{\mathfrak{f}}_{k}$, 
because the maximal number of components of $\overline{\mathrm{f}}_{j}, j=0,1,2, \ldots$ is bounded above. A condition for the existence of a solution of the problem (1.1) and (1.2) is $\operatorname{dim} \bar{f}_{k} \leq \operatorname{dim} \mathbf{y}$.

If there exists at least one solution $\mathbf{z}_{k}$ of the equation $\bar{f}_{k}\left(\mathbf{z}_{k}\right)=0$ which is a necessary condition, then from the equation $\overline{\mathbb{f}}_{k}(\mathbf{x}, \mathbf{y})=0$ by the implicit function theorem $\mathbf{y}$ can be expressed as a function of $\mathbf{x}$. This can be done as follows. The vector $\mathbf{y}$ can be partitioned as $\mathrm{y}=\left[\begin{array}{l}\mathrm{y}^{\prime} \\ \mathrm{y}^{\prime \prime}\end{array}\right]$ so that the matrix $\partial \overline{\mathrm{f}}_{k} / \partial \mathrm{y}^{\prime}$ is nonsingular. Thus the equation $\overline{\mathrm{f}}_{k}(\mathrm{x}, \mathrm{y})=0$ can be solved with respect to $\mathbf{y}^{\prime}, \mathbf{y}^{\prime}=\varphi\left(\mathbf{x}, \mathbf{y}^{\prime \prime}\right)$. Let us partition the PDE (1.1) in accordance with the partitioning of the vector $\mathbf{y}$, i.e.

$$
\frac{\partial \mathbf{y}^{\prime}}{\partial \mathbf{x}} \cdot \mathrm{h}_{i}(\mathrm{x}, \mathrm{y})+\mathrm{g}_{i}^{\prime}(\mathbf{x}, \mathbf{y})=0, \quad \frac{\partial \mathbf{y}^{\prime \prime}}{\partial \mathbf{x}} \cdot \mathrm{h}_{i}(\mathrm{x}, \mathrm{y})+\mathrm{g}_{i}^{\prime \prime}(\mathbf{x}, \mathbf{y})=0, \quad i=1, \ldots, q,
$$

where

$$
\left[\mathrm{g}_{1}, \ldots, \mathrm{g}_{q}\right]=\left[\begin{array}{lll}
\mathbf{g}_{1}^{\prime} & \cdots & \mathbf{g}_{q}^{\prime} \\
\mathbf{g}_{1}^{\prime \prime} & \cdots & \mathbf{g}_{q}^{\prime \prime}
\end{array}\right]
$$

and obtain a new system of PDEs with respect to $\mathbf{y}^{\prime \prime}$

$$
\frac{\partial \mathbf{y}^{\prime \prime}}{\partial \mathbf{x}} \cdot \mathbf{h}_{i}\left(\mathbf{x}, \varphi\left(\mathbf{x}, \mathbf{y}^{\prime \prime}\right), \mathbf{y}^{\prime \prime}\right)+\mathbf{g}_{i}^{\prime \prime}\left(\mathbf{x}, \varphi\left(\mathbf{x}, \mathbf{y}^{\prime \prime}\right), \mathbf{y}^{\prime \prime}\right)=0, \quad i=1, \ldots, q,
$$

and a system of algebraic equations

$$
\frac{\partial \varphi}{\partial \mathbf{x}} \cdot \mathbf{h}_{i}-\frac{\partial \varphi}{\partial \mathbf{y}^{\prime \prime}} \cdot \mathbf{g}_{i}^{\prime \prime}+\mathbf{g}_{i}^{\prime}=0, \quad i=1, \ldots, q,
$$

which does not give us anything new in solving of the problem (1.1) and (1.2) because it is equivalent to the equation $\overline{\mathbf{f}}_{k+1}=\overline{\mathbf{f}}_{k}$, i.e. $\tilde{\mathrm{f}}_{k+1}=0$.

Theorem 1.1. Sufficient conditions for the existence of a solution for the problem (1.1) and (1.2) are

i) $\operatorname{dim} \overline{\mathrm{f}}_{k} \leq \operatorname{dim} \mathrm{y}$,

ii) there exists a solution $\mathbf{z}_{k}$ of $\overline{\mathbf{f}}_{k}(\mathbf{z})=\mathbf{0}$,

iii) $\tilde{\mathfrak{f}}_{k+1}\left(\mathrm{z}_{k}\right)=0$,

iv) the new system of PDEs (1.8) is satisfied.

A solution of the problem is given by $\mathbf{y}_{k}^{\prime}$ and $\mathbf{y}_{k}^{\prime \prime}$, where the components of the vector $\mathbf{y}$ are partitioned (and eventually permuted) into the vectors $\mathrm{y}^{\prime}$ and $\mathrm{y}^{\prime \prime}$ so that the matrix $\partial \overline{\mathbf{f}}_{k} / \partial \mathrm{y}^{\prime}$ is nonsingular. Thus $\mathrm{y}_{k}^{\prime \prime}$ is a function which satisfies the system of PDEs (1.8) and $\mathrm{y}_{k}^{\prime}$ is a solution of $\overline{\mathbf{f}}_{k}\left(\mathbf{x}, \mathbf{y}_{k}\right)=\overline{\mathbf{f}}_{k}\left(\mathbf{x}, \mathbf{y}_{k}^{\prime}, \mathbf{y}_{k}^{\prime \prime}\right)=\mathbf{0}$.

According to this theorem we obtain again a system of PDEs (1.8) with dimension less than the former one and without algebraic constraints. Moreover, the following proposition holds. 
Proposition 1.2. If the system of PDEs (1.3) identically satisfies the compatibility conditions, then the system of PDEs (1.8) also identically satisfies the compatibility conditions.

The paper [5] is a special case of the previous results such that $f$ is a linear function of $\mathrm{y}, \mathrm{h}_{i}$ are functions of $\mathrm{x}$ only and $\mathrm{g}_{i}=\mathbf{0}$.

In the papers [7] and [8] solutions of systems of PDEs are given assuming that the systems are analytical. Let us consider the following system

$$
\frac{\partial \mathbf{y}}{\partial x_{u}}+\mathbf{G}_{u} \cdot \mathbf{y}=\mathbf{0} \quad(1 \leq u \leq n)
$$

where $\mathbf{y}=\left(y_{1}, \ldots, y_{m}\right)^{T}$ are unknown functions of $\mathbf{x}=\left(x_{1}, \ldots, x_{n}\right)^{T}$ and $\mathbf{G}_{u}$ are analytical matrix functions of $\mathbf{x}$. Then the compatibility conditions for (1.10) are

$$
\mathbb{R}_{i j} \equiv 0
$$

where

$$
\mathbb{R}_{i j}=\partial \mathbf{G}_{j} / \partial x_{i}-\partial \mathbf{G}_{i} / \partial x_{j}+\mathbf{G}_{i} \cdot \mathbf{G}_{j}-\mathbf{G}_{j} \cdot \mathbf{G}_{i} \quad(1 \leq i, j \leq n) .
$$

In [7] the following theorem is proved.

Theorem 1.3. Let the system (1.10) be given and let the compatibility conditions (1.11) be satisfied. Then there exist $m \times m$ matrix functions $\mathbb{P}^{\left\langle v_{1}, \ldots, v_{n}\right\rangle}(\mathrm{x}), v_{1}, \ldots, v_{n} \in$ $\mathrm{N}_{0}$ such that

$$
\begin{aligned}
\mathbb{P}^{<0, \ldots, 0\rangle} & =\mathbb{I} \\
\mathbb{P}^{\left\langle v_{1}, \ldots, v_{i}+1, \ldots, v_{n}\right\rangle} & =\frac{\partial}{\partial x_{i}} \mathbb{P}^{\left\langle v_{1}, \ldots, v_{n}\right\rangle}+\mathbf{G}_{i} \cdot \mathbb{P}^{\left\langle v_{1}, \ldots, v_{n}\right\rangle}
\end{aligned}
$$

and the solution of (1.10) is given by

$$
\mathrm{y}=\sum_{v_{1}=0}^{\infty} \sum_{v_{2}=0}^{\infty} \cdots \sum_{v_{n}=0}^{\infty} \frac{\left(-x_{1}\right)^{v_{1}}}{v_{1} !} \frac{\left(-x_{2}\right)^{v_{2}}}{v_{2} !} \cdots \frac{\left(-x_{n}\right)^{v_{n}}}{v_{n} !} \cdot \mathbb{P}^{\left.<v_{1}, \ldots, v_{n}\right\rangle} \cdot \mathrm{c},
$$

where $\mathrm{c}=\left(c_{1}, \ldots, c_{m}\right), c_{s}=y_{s}(\mathbf{0})$.

Further in [7] the nonlinear system of PDEs

$$
\frac{\partial \mathrm{y}}{\partial x_{u}}+\sum_{i_{1}, \ldots, i_{m} \in \mathbf{Z}} \mathbf{f}_{i_{1} \cdots i_{m} u}(\mathrm{x}) y_{1}^{i_{1}} y_{2}^{i_{2}} \cdot y_{m}^{i_{m}}=0 \quad(1 \leq u \leq n)
$$

is considered, where $\mathrm{f}_{i_{1} \ldots i_{m} u}$ are analytical functions. Moreover, suppose that there exists a neighbourhood $U$ of 0 such that all vector functions $\mathbf{f}_{i_{1} \cdots i_{m} u}$ are regular in $U$. Let $W$ be such that the Laurent series in (1.15) converge for $\mathbf{y} \in W$ and $\mathbf{x} \in U$. If $f_{r i_{1} \cdots i_{m} u}(\mathbf{x})$ 
$\left(1 \leq r \leq m, 1 \leq u \leq n, i_{1}, \ldots, i_{m} \in \mathbb{Z}\right)$ are the coordinates of $f_{i_{1} \ldots i_{m} u}$, we define new functions

$$
h_{i_{1} \cdots i_{m} j_{1} \cdot j_{m} u} \text { and } R_{i_{1} \cdots i_{m} j_{1} \cdot j_{m} u v} \quad\left(i_{1}, \ldots, i_{m}, j_{1}, \ldots, j_{m} \in \mathbb{Z}, 1 \leq u, v \leq n\right)
$$

by

$$
h_{i_{1} \cdot i_{m} j_{1} \cdots j_{m} u}=\sum_{s=1}^{m} i_{s} f_{s}\left(j_{1}-i_{1}\right) \cdots\left(j_{s}-i_{s}+1\right) \cdots\left(j_{m}-i_{m}\right) u
$$

and

$$
\begin{aligned}
& R_{i_{1} \cdots j_{m} j_{1} \cdots j_{m} u v}=\frac{\partial}{\partial x_{u}} h_{i_{1} \cdots i_{m} j_{1} \cdots j_{m} v}-\frac{\partial}{\partial x_{v}} h_{i_{1} \cdots i_{m} j_{1} \cdots j_{m} u} \\
& +\sum_{t_{1}, \ldots, t_{m} \in \mathbf{Z}} h_{t_{1} \cdots t_{m} j_{1} \cdots j_{m} v} h_{i_{1} \ldots i_{m} t_{1} \cdots t_{m} u}-\sum_{t_{1}, \ldots, t_{m} \in \mathbf{Z}} h_{t_{1} \cdots t_{m} j_{1} \cdots j_{m} u} h_{i_{1} \cdots i_{m} t_{1} \cdots t_{m} v} .
\end{aligned}
$$

Note that the series

$$
\sum_{j_{1}, \ldots, j_{m} \in \mathbb{Z}} h_{i_{1} \ldots i_{m} j_{1} \cdots j_{m} u} y_{1}^{j_{1}} \cdots y_{m}^{j_{m}} \text { and } \sum_{j_{1}, \ldots, j_{m} \in \mathbb{Z}} R_{i_{1} \cdots i_{m} j_{1} \cdots j_{m} u v} y_{1}^{j_{1}} \cdots y_{m}^{j_{m}}
$$

converge for $\mathrm{y} \in W$ and $\mathrm{x} \in U$. In order to simplify the notations, sometimes we will denote by the Greek indices $\alpha, \beta, \gamma, \ldots$ a set of $m$ integer indices $i_{1} \cdots i_{m} ; j_{1} \cdots j_{m} ; \cdots$ We will denote by $\{r\}$ the set of $m$ indices $0 \ldots 010 \ldots 0$ where the unit appears at the $r$-th place. Now $\alpha+\beta$ and $\alpha-\beta$ are defined by $i_{1} \cdots i_{m} \pm j_{1} \cdots j_{m}=\left(i_{1} \pm j_{1}\right)\left(i_{2} \pm\right.$ $\left.j_{2}\right) \cdots\left(i_{m} \pm j_{m}\right)$. The following properties are proved in [7]:

$$
\begin{aligned}
h_{(\alpha+\beta) \gamma u} & =h_{\alpha(\gamma-\beta) u}+h_{\beta\left(\gamma_{\alpha}\right) u}, \\
R_{(\alpha+\beta) \gamma u v} & =R_{\alpha(\gamma-\beta) u v}+R_{\beta(\gamma-\alpha) u v}, \\
h_{\alpha \beta u} & =\sum_{s=1}^{m} i_{s} h_{\{s\}(\beta-\alpha+\{s\}) u}, \\
R_{c . \beta u v} & =\sum_{s=1}^{m} i_{s} R_{\{s\}(\beta-\alpha+\{s\}) u v}, \text { where } \alpha=i_{1} \cdots i_{m} .
\end{aligned}
$$

Theorem 1.4. (i) The compatibility conditions for the system (1.15) for arbitrary initial conditions $\mathrm{y}(\mathbf{0})=\mathrm{c}$ are

$$
R_{\alpha \beta u v} \equiv 0, \text { i.e. } \quad R_{\{r\} \beta u v} \equiv 0 \text {. }
$$

(ii) If the compatibility conditions (1.22) are satisfied, then there exist functions

$$
P_{i_{1} \ldots i_{m} j_{1} \cdots j_{m}}^{<w_{1}, \ldots, w_{n}>}(\mathbf{x}), \quad w_{1}, \ldots, w_{n} \in \mathbb{N}_{0}, \quad i_{1}, \ldots, i_{m}, j_{1}, \ldots, j_{m} \in \mathbb{Z}
$$


in a neighbourhood of 0 such that

$$
\begin{aligned}
& P_{i_{1} \cdots i_{m} j_{1} \cdots j_{m}}^{<0, \ldots, 0>}=\delta_{i_{1} j_{1}} \delta_{i_{2} j_{2}} \cdots \delta_{i_{m} j_{m}}, \\
& P_{i_{1} \cdots i_{m} j_{1} \cdots j_{m}}^{<w_{1}, \ldots, w_{u}+1, \ldots, w_{n}>}=\frac{\partial}{\partial x_{u}} P_{i_{1} \cdots i_{m} j_{1} \cdots j_{m}}^{<w_{1}, \ldots, w_{n}>} \\
& \quad+\sum_{t_{1}, \ldots, i_{m} \in \mathbb{Z}}\left(\sum_{s=1}^{m} i_{s} f_{s\left(t_{1}-i_{1}\right) \cdots\left(t_{s}-i_{s}+1\right) \cdots\left(t_{m}-i_{m}\right) u}\right) P_{t_{1} \cdots t_{m} j_{1} \cdots j_{m}}^{<w_{1}, \ldots, w_{n}>}
\end{aligned}
$$

If $\mathrm{c} \in W$, then the solution of (1.15) in a neighbourhood of 0 is given by

$$
y_{r}=\sum_{w_{1}, \ldots, w_{n} \in \mathbb{N}_{0}}\left[\frac{\left(-x_{1}\right)^{w_{1}}}{w_{1} !} \cdots \frac{\left(-x_{n}\right)^{w_{n}}}{w_{n} !} \sum_{j_{1}, \ldots, j_{m} \in \mathbb{Z}} P_{\{r\} j_{1} j_{2} \cdots j_{m}}^{\left.<w_{1}, \ldots, w_{n}\right\rangle} c_{1}^{j_{1}} \cdot c_{2}^{j_{2}} \cdots c_{m}^{j_{m}}\right]
$$

for $1 \leq r \leq m$. This solution is unique with the given initial conditions in a neighbourhood. of 0 .

The paper [8] generalizes the paper [7] so that for the corresponding system the compatibility conditions are not assumed identically satisfied and they depend on the choice of the initial conditions. If the initial conditions are given, then the necessary and sufficient conditions for the existence of a solution are given, and if they are satisfied, the solution is given in a functional series as in Theorems 1.3 and 1.4.

\section{Solution of the Cauchy Problem}

In this section we will give the solution of the Cauchy problem for linear and nonlinear systems of PDEs. Let $p \leq n$ and let us consider the following system

$$
\partial \mathbf{y} / \partial x_{u}+\mathbb{G}_{u} \cdot \mathbf{y}=\mathbf{0} \quad(u=1, \ldots, p)
$$

with the initial condition

$$
\mathrm{y}\left(0, \ldots, 0, x_{p+1}, \ldots, x_{n}\right)=\psi\left(\dot{x_{p+1}}, \ldots, x_{n}\right)
$$

with respect to the unknown vector function $\mathbf{y}=\left(y_{1}, \ldots, y_{m}\right)$ of the vector variable $\mathrm{x}=\left(x_{1}, \ldots, x_{n}\right)$, where $\mathbb{G}_{u}$ are given analytical matrix functions of $\mathbf{x}$ regular in a neighbourhood of $\left(0, \ldots, 0, z_{p+1}, \ldots, z_{n}\right)$ and $\psi$ is a given vector function. The compatibility conditions for the system (2.1) with arbitrary initial conditions (2.2) are

$$
\mathbb{R}_{i j} \equiv 0 \quad(i, j=1, \ldots, p)
$$

where $\mathbf{R}_{i j}$ were defined in Section 1 . Now we will prove the following theorem.

Theorem 2.1. Let the system (2.1) with the initial conditions (2.2) be given and the compatibility conditions (2.3) be satisfied. Then there exist $m \times m$ matrix functions 
$\mathbb{P}^{\left\langle w_{1}, \ldots, w_{p}\right\rangle}(\mathbf{x}), w_{1}, \ldots, w_{p} \in \mathbb{N}_{0}$ such that

$$
\begin{gathered}
\mathbb{P}^{<0, \ldots, 0\rangle}=\mathbb{I} \\
\mathbb{P}^{\left\langle w_{1}, \ldots, w_{u}+1, \ldots, w_{p}\right\rangle}=\frac{\partial}{\partial x_{u}} \mathbb{P}^{\left\langle w_{1}, \ldots, w_{p}\right\rangle}+\mathbb{G}_{u} \cdot \mathbb{P}^{\left.<w_{1}, \ldots, w_{u}, \ldots, w_{p}\right\rangle}, \quad u=1, \ldots, p,
\end{gathered}
$$

and the solution of (2.1) with (2.2) in a neighbourhood of $\left(0, \ldots, 0, z_{p+1}, \ldots, z_{n}\right)$ is given by

$$
\mathrm{y}=\sum_{w_{1}=0}^{\infty} \sum_{w_{2}=0}^{\infty} \cdots \sum_{w_{p}=0}^{\infty} \frac{\left(-x_{1}\right)^{w_{1}}}{w_{1} !} \frac{\left(-x_{2}\right)^{w_{2}}}{w_{2} !} \cdots \frac{\left(-x_{p}\right)^{w_{p}}}{w_{p} !} \times \mathbb{P}^{\left.<w_{1}, \ldots, w_{p}\right\rangle} \cdot \psi\left(x_{p+1} \ldots, x_{k}\right) .
$$

This solution is unique with the given initial conditions in a neighbourhood of $(0, \ldots, 0$, $\left.z_{p+1}, \ldots, z_{n}\right)$.

Proof. Suppose that the system (2.1) is given and the compatibility conditions (2.3) are satisfied. In order to prove that there exist matrix functions

$$
\mathbb{P}^{<w_{1}, \ldots, w_{p}>}(\mathbf{x}) \quad\left(w_{1}, \ldots, w_{p} \in \mathbf{N}_{0}\right)
$$

such that $(2.4 \mathrm{a})$ and $(2.4 \mathrm{~b})$ are satisfied, it is sufficient to prove that

$$
\mathbb{P}^{\left\langle w_{1}, \ldots, w_{u}^{(2)}+1, \ldots, w_{v}^{(1)}+1, \ldots, w_{p}\right\rangle}=\mathbb{P}^{\left\langle w_{1}, \ldots, w_{u}^{(1)}+1, \ldots, w_{v}^{(2)}+1, \ldots, w_{p}\right\rangle}
$$

for each $u, v \in\{1, \ldots, p\}, u<v$, where $w_{u}^{(1)}=w_{v}^{(1)}, w_{u}^{(2)}=w_{v}^{(2)}$. Indeed, using the definition of the matrix functions $\mathbb{P}$ and the compatibility condition (2.3), we obtain

$$
\begin{aligned}
& \mathbb{P}^{\left.<w_{1}, \ldots w_{u}^{(2)}+1, \ldots w_{v}^{(1)}+1, \ldots w_{p}\right\rangle}-\mathbb{P}^{\left\langle w_{1}, \ldots, w_{u}^{(1)}+1, \ldots w_{v}^{(2)}+1, \ldots w_{p}\right\rangle} \\
= & \mathbb{R}_{u v} \cdot \mathbb{P}^{\left.<w_{1}, \ldots, w_{p}\right\rangle} \equiv \mathbf{0} .
\end{aligned}
$$

Now we should prove that the vector function y defined by (2.5) satisfies the system (2.1) and the initial condition (2.2). The uniform convergence of the right-hand side of (2.5) in a neighbourhood of $\mathbf{0}$ is proved in [7]. Further, for an arbitrary vector function $\psi\left(x_{p+1}, \ldots, x_{n}\right)$ according to $(2.5)$ and $(2.4 \mathrm{~b})$ we obtain

$$
\begin{aligned}
\partial \mathrm{y} / \partial x_{u}= & \sum_{w_{1}, \ldots, w_{p} \in \mathbf{N}_{0}} \frac{\left(-x_{1}\right)^{w_{1}}}{w_{1} !} \cdots(-1) \frac{\left(-x_{u}\right)^{w_{u}-1}}{\left(w_{u}-1\right) !} \cdots \frac{\left(-x_{p}\right)^{w_{p}}}{w_{p} !} \\
& \times \mathbb{P}^{<w_{1}, \ldots, w_{p}>} \cdot \psi\left(x_{p+1}, \ldots, x_{n}\right) \\
& +\sum_{w_{1}, \ldots, w_{p} \in \mathbf{N}_{0}} \frac{\left(-x_{1}\right)^{w_{1}}}{w_{1} !} \cdots \frac{\left(-x_{p}\right)^{w_{p}}}{w_{p} !} \cdot \frac{\partial}{\partial x_{u}}\left[\mathbb{P}^{<w_{1}, \ldots, w_{p}>} \cdot \psi\left(x_{p+1}, \ldots, x_{n}\right)\right] \\
= & -\sum_{\substack{w_{1}, \ldots, w_{p} \in \mathbf{N}_{0}\\
}} \frac{\left(-x_{1}\right)^{w_{1}}}{w_{1} !} \cdots \frac{\left(-x_{u}\right)^{w_{u}}}{w_{u} !} \cdots \frac{\left(-x_{p}\right)^{w_{p}}}{w_{p} !} \\
& \times \mathbb{P}^{<w_{1}, \ldots, w_{u+1}, \ldots, w_{p}>} \cdot \psi\left(x_{p+1}, \ldots, x_{n}\right)
\end{aligned}
$$




$$
\begin{aligned}
& +\sum_{w_{1}, \ldots, w_{p} \in \mathbf{N}_{0}} \frac{\left(-x_{1}\right)^{w_{1}}}{w_{1} !} \cdots \frac{\left(-x_{p}\right)^{w_{p}}}{w_{p} !} \cdot\left[\frac{\partial}{\partial x_{u}} \mathbb{P}^{\left.<w_{1}, \ldots, w_{p}\right\rangle}\right] \cdot \psi\left(x_{p+1}, \ldots, x_{n}\right) \\
= & -\sum_{w_{1}, \ldots, w_{p} \in \mathbf{N}_{0}} \frac{\left(-x_{1}\right)^{w_{1}}}{w_{1} !} \cdots \frac{\left(-x_{p}\right)^{w_{p}}}{w_{p} !} \cdot\left[\mathbb{P}^{\left.<w_{1}, \ldots, w_{u}+1, \ldots, w_{p}\right\rangle}\right. \\
& \left.-\frac{\partial}{\partial x_{u}} \mathbb{P}^{<w_{1}, \ldots, w_{p}>}\right] \cdot \psi\left(-x_{p+1}, \ldots, x_{n}\right) \\
= & -\sum_{w_{1}, \ldots, w_{p} \in \mathbf{N}_{0}} \frac{\left(-x_{1}\right)^{w_{1}}}{w_{1} !} \cdots \frac{\left(-x_{p}\right)^{w_{p}}}{w_{p} !} \cdot\left[\mathbf{G}_{u} \cdot \mathbb{P}^{\left.<w_{1}, \ldots, w_{p}\right\rangle}\right] \cdot \psi\left(x_{p+1}, \ldots x_{n}\right) \\
= & -\mathbf{G}_{u} \cdot\left[\sum_{w_{1}, \ldots, w_{p} \in \mathbf{N}_{0}} \frac{\left(-x_{1}\right)^{w_{1}}}{w_{1} !} \cdots \frac{\left(-x_{p}\right)^{w_{p}}}{w_{p} !} \cdot \mathbb{P}^{\left.<w_{1}, \ldots, w_{p}\right\rangle} \cdot \psi\left(x_{p+1}, \ldots, x_{n}\right)\right] \\
= & -\mathbf{G}_{u} \cdot \mathbf{y},
\end{aligned}
$$

and hence (2.1) is satisfied. Further, according to (2.4a) we obtain

$$
\mathrm{y}(0)=\mathbb{P}^{<0, \ldots, 0>} \cdot \psi\left(x_{p+1}, \ldots, x_{n}\right)=\mathbb{I} \cdot \psi\left(x_{p+1}, \ldots, x_{n}\right)=\psi\left(x_{p+1}, \ldots, x_{n}\right) .
$$

The solution is unique with the given initial coditions. This is proved analogously to [7].

Let us consider the following nonlinear system of PDEs

$$
\partial \mathrm{y} / \partial x_{u}+\sum_{i_{1}, \ldots i_{m} \in \mathbb{Z}} \mathbb{f}_{i_{1} \cdots i_{m} u}(\mathbf{x}) y_{1}^{i_{1}} y_{2}^{i_{2}} \cdots y_{m}^{i_{m}}=0, \quad u=1, \ldots, p \quad(p \leq n)
$$

with the initial condition

$$
\mathbf{y}\left(0, \ldots, 0, x_{p+1}, \ldots, x_{n}\right)=\psi\left(x_{p+1}, \ldots, x_{n}\right),
$$

where $\mathrm{f}_{i_{1} \cdots i_{m} u}=\left(f_{1 i_{1} \cdots i_{m} u}, \ldots, f_{m i_{1} \cdots i_{m} u}\right)$ are analytical functions and suppose that there exists a neighbourhood $U$ of $\left(0, \ldots, 0, z_{p+1}, \ldots, z_{n}\right)$ such that all functions $f_{r i_{1}, \ldots i_{m} u}$ are regular in $U$. Let $W$ be such that the Laurent series in (2.7) converge for $\mathbf{y} \in W$ and $\mathrm{x} \in U$, analogously as in Section 1 . We define the quantities $R_{\alpha \beta u v}$ in the same way as in Section 1 and now we have the following theorem.

Theorem 2.2. (i) The compatibility conditions for the system (2.7). for arbitrary initial conditions (2.8) are

$$
R_{\alpha \beta u v} \equiv 0, \quad \text { i.e. } \quad R_{\{r\} \beta u v} \equiv 0, \quad u, v=1, \ldots, p .
$$

(ii) If the compatibility conditions (2.9) are satisfied, then there exist functions

$$
P_{i_{1} \cdots i_{m} j_{1} \cdots j_{m}}^{<w_{1}, \ldots w_{p}>}(\mathbf{x}), \quad w_{1}, \ldots, w_{p} \in \mathbb{N}_{0}, \quad i_{1}, \ldots i_{m}, j_{1}, \ldots, j_{m} \in \mathbb{Z}
$$

in a neighbourhood of $\left(0, \ldots, 0, z_{p+1}, \ldots, z_{n}\right)$ such that

$$
\begin{aligned}
& P_{i_{1} \ldots i_{m} j_{1} \cdots j_{m}}^{<0, \ldots, 0>}=\delta_{i_{1} j_{1}} \delta_{i_{2} j_{2}} \cdots \delta_{i_{m} j_{m}}, \\
& P_{i_{1} \cdots i_{m} j_{1} \cdots j_{m}}^{<w_{1}, \ldots, w_{u}+1, \ldots, w_{p}>}=\frac{\partial}{\partial x_{u}} P_{i_{1} \cdots i_{m} j_{1} \cdots j_{m}}^{<w_{1}, \ldots, w_{p}>} \\
& \quad+\sum_{t_{1}, \ldots, t_{m} \in \mathbb{Z}}\left(\sum_{s=1}^{m} i_{s} f_{s\left(t_{1}-i_{1}\right) \cdots\left(t_{s}-i_{s}+1\right) \cdot\left(t_{m}-i_{m}\right) u}\right) p_{t_{1} \cdots t_{m} j_{1} \cdots j_{m}}^{<w_{1}, \ldots, w_{p}>} .
\end{aligned}
$$


If $\psi\left(x_{p+1}, \ldots, x_{n}\right) \in W$, then the solution of (2.7) and (2.8) in a neighbourhood of $\left(0, \ldots, 0, z_{p+1}, \ldots, z_{n}\right)$ is given by

$$
y_{r}=\sum_{w_{1}, \ldots, w_{p} \in \mathbf{N}_{0}}\left[\frac{\left(-x_{1}\right)^{w_{1}}}{w_{1} !} \cdots \frac{\left(-x_{p}\right)^{w_{p}}}{w_{p} !} \sum_{j_{1}, \ldots, j_{m} \in \mathbf{Z}} P_{\left\{r j_{1} \cdots j_{m}\right.}^{<w_{1}, \ldots, w_{p}>} \psi_{1}^{j_{1}} \cdot \psi_{2}^{j_{2}} \cdots \psi_{m}^{j_{m}}\right] .
$$

This solution is unique with the given initial conditions in a neighborhood of $(0, \ldots, 0$, $\left.z_{p+1}, \ldots, z_{n}\right)$.

If we introduce the variables

$$
y_{\alpha}=y_{i_{1} i_{2} \cdots i_{m}}=y_{1}^{i_{1}} \cdot y_{2}^{i_{2}} \cdots y_{m}^{i_{m}}, \quad\left(i_{1}, \ldots, i_{m} \in \mathbb{Z}\right),
$$

then the nonlinear system (2.7) is transformed into an infinitely dimensional linear system of PDEs with unknown functions $y_{\alpha}, \alpha \in \mathbb{Z}^{n}$. The proof of Theorem 2.2 is analogous to the proof of Theorem 2.1 (see also [7, Theorem 3.2]).

\section{Reduction of a System of Partial Differential Equations}

In this section we return to [6], considering the algorithm for reduction of PDEs and the problem about the compatibility conditions for the derived system of PDEs with linear homogeneous algebraic constraints (see Theorem 1.1 and Proposition 1.2):

$$
\mathfrak{f}(\mathrm{x}, \mathrm{y})=\mathbb{F}(\mathbf{x}) \cdot \mathbf{y}
$$

Let us consider the following homogeneous linear system of PDEs

$$
\frac{\partial \mathbf{y}}{\partial x_{i}}+\mathbf{G}_{i} \cdot \mathbf{y}=0, \quad i=1, \ldots, p(p \leq n)
$$

Let $\mathrm{f}_{0}(\mathrm{x}, \mathrm{y})=\mathrm{F}_{0}(\mathrm{x}) \cdot \mathrm{y}$ for a matrix function $\mathbb{F}_{0}(\mathrm{x})$ be the vector function obtained by uniting the given algebraic constraints and algebraic constraints concerning the compatibility conditions:

$$
\left[\frac{\partial \mathbf{G}_{i}}{\partial x_{j}}-\mathbf{G}_{i} \cdot \mathbf{G}_{j}-\frac{\partial G_{j}}{\partial x_{i}}+\mathbf{G}_{j} \cdot \mathbf{G}_{\mathbf{i}}\right] \cdot \mathbf{y}=\mathbf{0} \quad(1 \leq i, j \leq p) .
$$

By applying the reduction algorithm (see Section 1) we obtain sucessively the functions

$$
\mathrm{f}_{0}(\mathrm{x}, \mathrm{y})=\mathbb{F}_{0}(\mathrm{x}) \cdot \mathrm{y}, \quad \mathbf{f}_{1}(\mathrm{x}, \mathrm{y})=\mathbb{F}_{1}(\mathrm{x}) \cdot \mathrm{y}, \ldots, \mathrm{f}_{k}(\mathrm{x}, \mathrm{y})=\mathbb{F}_{\mathbf{k}}(\mathrm{x}) \cdot \mathrm{y} .
$$

Let us partition the functions $\mathbb{F}_{i}$ as

$$
\mathbb{F}_{i}=\left[\begin{array}{c}
\overline{\mathrm{F}}_{i} \\
\tilde{\mathrm{F}}_{i}
\end{array}\right]
$$


where the rows of $\overline{\mathbf{F}}_{i}$ are linearly independent, and the rows of $\tilde{\mathbf{F}}_{i}$ are linearly dependent on the rows of $\overrightarrow{\mathbb{F}}_{i}$. According to the definition of the final index $k$ there exist matrix functions $\mathrm{V}_{i}$ such that

$$
\frac{\partial \overline{\mathbf{F}}_{k}}{\partial x_{i}}-\overline{\mathbf{F}}_{k} \cdot \mathbb{G}_{i}=\mathbf{V}_{i} \cdot \overline{\mathbb{F}}_{k}
$$

Thus by differentiating the following equation

$$
\overline{\mathfrak{f}}_{k}(\mathbf{x}, \mathbf{y})=\overline{\mathbf{F}}_{k}(\mathbf{x}) \cdot \mathbf{y}=\mathbf{0}
$$

with respect to $x_{i}$ we do not obtain new constraints. The last equality can be written in the following form:

$$
\left[\overline{\mathbb{F}}_{k}^{\prime} \overline{\mathbf{F}}_{k}^{\prime \prime}\right] \cdot\left[\begin{array}{l}
\mathrm{y}^{\prime} \\
\mathbf{y}^{\prime \prime}
\end{array}\right]=\overline{\mathbf{F}}_{k}^{\prime} \cdot \mathbf{y}^{\prime}+\overline{\mathbf{F}}_{k}^{\prime \prime} \cdot \mathbf{y}^{\prime \prime}=0, \quad \mathbf{y}=\left[\begin{array}{l}
\mathrm{y}^{\prime} \\
\mathbf{y}^{\prime \prime}
\end{array}\right],
$$

where $\overline{\mathrm{F}}_{k}^{\prime}$ is a nonsingular matrix. Hence,

$$
\mathbf{y}^{\prime}=-\overline{\mathbb{F}}_{k}^{\prime-1} \cdot \overline{\mathbf{F}}^{\prime \prime} \cdot \mathrm{y}^{\prime \prime} .
$$

The system (3.1) decomposes into

$$
\begin{aligned}
& \frac{\partial \mathbf{y}^{\prime}}{\partial x_{i}}+\overline{\mathbf{G}}_{i}^{\prime} \cdot \mathbf{y}^{\prime}+\overline{\mathbf{G}}_{i}^{\prime \prime} \cdot \mathbf{y}^{\prime \prime}=0 \\
& \frac{\partial \mathbf{y}^{\prime \prime}}{\partial x_{i}}+\tilde{\mathbf{G}}_{i}^{\prime} \cdot \mathbf{y}^{\prime}+\tilde{\mathbf{G}}_{i}^{\prime \prime} \cdot \mathbf{y}^{\prime \prime}=\mathbf{0}
\end{aligned}
$$

Now we prove that the equation (3.3) is a consequence of (3.4). First note that

$$
\begin{aligned}
& \frac{\partial}{\partial x_{i}}\left(\overline{\mathbf{F}}_{k}^{\prime-1} \cdot \overline{\mathbf{F}}_{k}^{\prime \prime}\right)=-\overline{\mathbf{F}}_{k}^{\prime-1} \cdot \frac{\partial \overline{\mathbf{F}}_{k}^{\prime}}{\partial x_{i}} \cdot \overline{\mathbf{F}}_{k}^{\prime-1} \cdot \overline{\mathbf{F}}_{k}^{\prime \prime}+\overline{\mathbb{F}}_{k}^{\prime-1} \cdot \frac{\partial \overline{\mathbb{F}}_{k}^{\prime \prime}}{\partial x_{i}} \\
& =-\overline{\mathbb{F}}_{k}^{\prime-1} \cdot\left(\mathbb{V}_{i} \cdot \overline{\mathbb{F}}_{k}^{\prime}+\overline{\mathbb{F}}_{k} \cdot \overline{\mathbf{G}}_{i}^{\prime}\right) \cdot \overline{\mathbf{F}}_{k}^{-1} \cdot \overline{\mathbb{F}}_{k}^{\prime \prime}+\overline{\mathbb{F}}_{k}^{\prime-1} \cdot\left(\mathbb{V}_{i} \cdot \overline{\mathbb{F}}_{k}^{\prime \prime}+\overline{\mathbb{F}}_{k} \cdot \mathbb{G}_{i}^{\prime \prime}\right) \\
& =\overline{\mathbb{F}}_{k}^{\prime-1} \cdot \overline{\mathrm{F}}_{k} \cdot\left(\mathbb{G}_{i}^{\prime \prime}-\mathbb{G}_{i}^{\prime} \cdot \overline{\mathbb{F}}_{k}^{\prime-1} \cdot \overline{\mathbb{F}}_{k}^{\prime \prime}\right) \text {, }
\end{aligned}
$$

where we have used the partitioned identity (3.2), i.e.

$$
\begin{aligned}
& \frac{\partial \overline{\mathbf{F}}_{k}^{\prime}}{\partial x_{i}}-\overline{\mathbf{F}}_{k} \cdot \mathbb{G}_{i}^{\prime}=\mathbb{V}_{i} \cdot \overline{\mathbf{F}}_{k}^{\prime} \\
& \frac{\partial \overline{\mathbf{F}}_{k}^{\prime \prime}}{\partial x_{i}}-\overline{\mathbf{F}}_{k} \cdot \mathbb{G}_{i}^{\prime \prime}=\mathbb{V}_{i} \cdot \overline{\mathbf{F}}_{k}^{\prime \prime}
\end{aligned}
$$

Further, for (3.3) we obtain

$$
\begin{aligned}
& \frac{\partial \mathrm{y}^{\prime}}{\partial x_{i}}+\overline{\mathrm{G}}_{i} \cdot \mathrm{y}^{\prime}+\overline{\mathrm{G}}_{i}^{\prime \prime} \cdot \mathrm{y}^{\prime \prime} \\
& =-\frac{\partial}{\partial x_{i}}\left(\overline{\mathbf{F}}_{k}^{\prime-1} \cdot \overline{\mathbb{F}}_{k}^{\prime \prime}\right) \cdot \mathbf{y}^{\prime \prime}-\overline{\mathbb{F}}_{k}^{\prime-1} \cdot \mathbb{F}_{k}^{\prime \prime} \cdot \frac{\partial \mathbf{y}^{\prime \prime}}{\partial x_{i}}+\left(\overline{\mathbf{G}}_{i}^{\prime \prime}-\overline{\mathbf{G}}_{i}^{\prime} \cdot \overline{\mathbf{F}}_{k}^{\prime-1} \cdot \overline{\mathbf{F}}_{k}^{\prime \prime}\right) \cdot \mathbf{y}^{\prime \prime} \\
& =-\overline{\mathbb{F}}_{k}^{\prime-1} \cdot \mathbb{F}_{k} \cdot\left(\mathbb{G}_{i}^{\prime \prime}-\mathbb{G}_{i}^{\prime} \cdot \overline{\mathbb{F}}_{k}^{\prime-1} \cdot \overline{\mathbb{F}}_{k}^{\prime \prime}\right) \cdot \mathbf{y}^{\prime \prime}-\overline{\mathbf{F}}_{k}^{\prime-1} \cdot \overline{\mathbb{F}}_{k}^{\prime \prime} \cdot \frac{\partial \mathbf{y}^{\prime \prime}}{\partial x_{i}}+\left(\overline{\mathbf{G}}_{i}^{\prime \prime}-\overline{\mathbf{G}}_{i}^{\prime} \cdot \overline{\mathbb{F}}_{k}^{\prime-1} \cdot \overline{\mathbf{F}}_{k}^{\prime \prime}\right) \cdot \mathbf{y}^{\prime \prime} \\
& =-\overline{\mathbf{F}}_{k}^{\prime-1} \cdot \overline{\mathbf{F}}_{k}^{\prime \prime}\left(\frac{\partial \mathbf{y}^{\prime \prime}}{\partial x_{i}}+\left(\tilde{\mathbf{G}}_{i}^{\prime \prime}-\tilde{\mathbf{G}}_{i}^{\prime} \cdot \overline{\mathbf{G}}_{k}^{\prime-1} \cdot \overline{\mathbf{F}}_{k}^{\prime \prime}\right) \cdot \mathbf{y}^{\prime \prime}\right)=0
\end{aligned}
$$


under the condition (3.4), i.e.

$$
\frac{\partial \mathbf{y}^{\prime \prime}}{\partial x_{i}}+\left(\tilde{\mathbf{G}}_{i}^{\prime \prime}-\tilde{\mathbf{G}}_{i}^{\prime} \cdot \overline{\mathbf{F}}_{k}^{\prime-1} \cdot \overline{\mathbf{F}}_{k}^{\prime \prime}\right) \cdot \mathbf{y}^{\prime \prime}=\mathbf{0}
$$

Thus we obtain a new system of PDEs with the same form as the former one but with a smaller dimension. Now we are ready to prove the main theorem.

Theorem 3.1. The compatibility conditions for the system of PDEs (3.5) are identically satisfied.

Proof. By the definition of $\mathbb{F}_{0}$ there exist matrices $\mathbb{V}_{i j}$ such that

$$
\frac{\partial \mathbf{G}_{i}}{\partial x_{j}}-\frac{\partial \mathbf{G}_{j}}{\partial x_{i}}-\mathbb{G}_{i} \mathbf{G}_{j}+\mathbb{G}_{j} \mathbf{G}_{i}=\mathbb{V}_{i j} \cdot \overline{\mathbf{F}}_{k}
$$

This equality decomposes into the following two equalities

$$
\begin{gathered}
\frac{\partial \mathbb{G}_{i}^{\prime}}{\partial x_{j}}-\frac{\partial \mathbb{G}_{j}^{\prime}}{\partial x_{i}}-\mathbb{G}_{i} \mathbb{G}_{j}^{\prime}+\mathbb{G}_{j} \mathbb{G}_{i}^{\prime}=\mathbb{V}_{i j} \cdot \overline{\mathbf{F}}_{k}^{\prime}, \\
\frac{\partial \mathbb{G}_{i}^{\prime \prime}}{\partial x_{j}}-\frac{\partial \mathbb{G}_{j}^{\prime \prime}}{\partial x_{i}}-\mathbb{G}_{i} \mathbb{G}_{j}^{\prime \prime}+\mathbb{G}_{j} \mathbb{G}_{i}^{\prime \prime}=\mathbb{V}_{i j} \cdot \overline{\mathbb{F}}_{k}^{\prime \prime}
\end{gathered}
$$

By elimination of $\mathrm{V}_{i j}$ we obtain

$$
\begin{aligned}
& \frac{\partial \mathbb{G}_{i}^{\prime \prime}}{\partial x_{j}}-\frac{\partial \mathbb{G}_{i}^{\prime}}{\partial x_{i}} \cdot \overline{\mathbb{F}}_{k}^{\prime-1} \cdot \overline{\mathbb{F}}_{k}^{\prime \prime}-\mathbb{G}_{i}\left(\mathbb{G}_{j}^{\prime \prime}-\mathbb{G}_{j}^{\prime} \cdot \overline{\mathbb{F}}_{k}^{\prime-1} \cdot \overline{\mathbb{F}}_{k}^{\prime \prime}\right) \\
= & \frac{\partial \mathbb{G}_{j}^{\prime \prime}}{\partial x_{j}}-\frac{\partial \mathbb{G}_{j}^{\prime}}{\partial x_{i}} \cdot \overline{\mathbb{F}}_{k}^{\prime-1} \cdot \overline{\mathbb{F}}_{k}^{\prime \prime}-\mathbb{G}_{j}\left(\mathbb{G}_{i}^{\prime \prime}-\mathbb{G}_{i}^{\prime} \cdot \overline{\mathbb{F}}_{k}^{\prime-1} \cdot \overline{\mathbb{F}}_{k}^{\prime \prime}\right) .
\end{aligned}
$$

Using the identity

$$
\frac{\partial}{\partial x_{j}}\left(\mathbb{G}_{i}^{\prime \prime}-\mathbb{G}_{i}^{\prime} \cdot \overline{\mathbb{F}}_{k}^{\prime-1} \cdot \overline{\mathbb{F}}_{k}^{\prime \prime}\right)=\frac{\partial \mathbb{G}_{i}^{\prime \prime}}{\partial x_{j}}-\frac{\partial \mathbb{G}_{i}^{\prime}}{\partial x_{j}} \cdot \overline{\mathbb{F}}^{\prime-1} \cdot \overline{\mathbb{F}}_{k}^{\prime \prime}-\mathbb{G}_{i}^{\prime} \cdot \overline{\mathbb{F}}_{k}^{\prime-1} \cdot \overline{\mathbb{F}}_{k} \cdot\left(\mathbb{G}_{j}^{\prime \prime}-\mathbb{G}_{j}^{\prime} \cdot \overline{\mathbb{F}}_{k}^{\prime-1} \cdot \overline{\mathbb{F}}_{k}^{\prime \prime}\right),
$$

from (3.6) we obtain

$$
\begin{aligned}
& \frac{\partial}{\partial x_{j}}\left(\mathbb{G}_{i}^{\prime \prime}-\mathbb{G}_{i}^{\prime} \cdot \overline{\mathbb{F}}_{k}^{\prime-1} \cdot \overline{\mathbb{F}}_{k}^{\prime \prime}\right)+\mathbb{G}_{i}^{\prime} \cdot \overline{\mathbb{F}}_{k}^{\prime-1} \cdot \overline{\mathbb{F}}_{k} \cdot\left(\mathbb{G}_{j}^{\prime \prime}-\mathbb{G}_{j}^{\prime} \cdot \overline{\mathbb{F}}_{k}^{\prime-1} \cdot \overline{\mathbb{F}}_{k}^{\prime \prime}\right) \\
& -\mathrm{G}_{i}\left(\mathrm{G}_{j}^{\prime \prime}-\mathbb{G}_{j}^{\prime} \cdot \overline{\mathbf{F}}_{k}^{\prime-1} \cdot \overline{\mathbf{F}}_{k}^{\prime \prime}\right) \\
& =\frac{\partial}{\partial x_{i}}\left(\mathbb{G}_{j}^{\prime \prime}-\mathbb{G}_{j}^{\prime} \cdot \overline{\mathbb{F}}_{k}^{\prime-1} \cdot \overline{\mathbb{F}}_{k}^{\prime \prime}\right)+\mathbb{G}_{j}^{\prime} \cdot \overline{\mathbb{F}}_{k}^{\prime-1} \cdot \overline{\mathbb{F}}_{k} \cdot\left(\mathbb{G}_{i}^{\prime \prime}-\mathbb{G}_{i}^{\prime} \cdot \overline{\mathbb{F}}_{k}^{\prime-1} \cdot \overline{\mathbb{F}}_{k}^{\prime \prime}\right) \\
& -\mathrm{G}_{j}\left(\mathrm{G}_{i}^{\prime \prime}-\mathrm{G}_{i}^{\prime} \cdot \overline{\mathbb{F}}_{k}^{\prime-1} \cdot \overline{\mathbb{F}}_{k}^{\prime \prime}\right) \text {, } \\
& \frac{\partial}{\partial x_{j}}\left(\mathbb{G}_{i}^{\prime \prime}-\mathbb{G}_{i}^{\prime} \cdot \overline{\mathbb{F}}_{k}^{\prime-1} \cdot \overline{\mathbb{F}}_{k}^{\prime \prime}\right)-\left(\mathbb{G}_{i}-\mathbb{G}_{i}^{\prime} \cdot \overline{\mathbb{F}}_{k}^{\prime-1} \cdot \overline{\mathbb{F}}_{k}^{\prime \prime}\right) \cdot\left(\mathbb{G}_{j}^{\prime \prime}-\mathbb{G}_{j}^{\prime} \cdot \overline{\mathbb{F}}_{k}^{\prime-1} \cdot \overline{\mathbb{F}}_{k}^{\prime \prime}\right)
\end{aligned}
$$




$$
\begin{aligned}
= & \frac{\partial}{\partial x_{i}}\left(\mathbb{G}_{j}^{\prime \prime}-\mathbb{G}_{j}^{\prime} \cdot \overline{\mathbb{F}}_{k}^{\prime-1} \cdot \overline{\mathbb{F}}_{k}^{\prime \prime}\right)-\left(\mathbb{G}_{j}-\mathbb{G}_{j}^{\prime} \cdot \overline{\mathbb{F}}_{k}^{\prime-1} \cdot \overline{\mathbb{F}}_{k}^{\prime \prime}\right) \cdot\left(\mathbb{G}_{i}^{\prime \prime}-\mathbb{G}_{i}^{\prime} \cdot \overline{\mathbb{F}}_{k}^{\prime-1} \cdot \overline{\mathbb{F}}_{k}^{\prime \prime}\right), \\
& \frac{\partial}{\partial x_{j}}\left(\mathbb{G}_{i}^{\prime \prime}-\mathrm{G}_{i}^{\prime} \cdot \overline{\mathbb{F}}_{k}^{\prime-1} \cdot \overline{\mathbb{F}}_{k}^{\prime \prime}\right)-\left(\mathbb{G}_{i}^{\prime \prime}-\mathbb{G}_{i}^{\prime} \cdot \overline{\mathbb{F}}_{k}^{\prime-1} \cdot \overline{\mathbb{F}}_{k}^{\prime \prime}\right) \cdot\left(\tilde{\mathbb{G}}_{j}^{\prime \prime}-\tilde{\mathbb{G}}_{j}^{\prime} \cdot \overline{\mathbb{F}}_{k}^{\prime-1} \cdot \overline{\mathbb{F}}_{k}^{\prime \prime}\right) \\
= & \frac{\partial}{\partial x_{i}}\left(\mathbb{G}_{j}^{\prime \prime}-\mathrm{G}_{j}^{\prime} \cdot \overline{\mathbb{F}}_{k}^{\prime-1} \cdot \overline{\mathbb{F}}_{k}^{\prime \prime}\right)-\left(\mathbb{G}_{j}^{\prime \prime}-\mathbb{G}_{j}^{\prime} \cdot \overline{\mathbb{F}}_{k}^{\prime-1} \cdot \overline{\mathbb{F}}_{k}^{\prime \prime}\right) \cdot\left(\tilde{\mathbb{G}}_{i}^{\prime \prime}-\tilde{\mathbb{G}}_{i}^{\prime} \cdot \overline{\mathbb{F}}_{k}^{\prime-1} \cdot \overline{\mathbb{F}}_{k}^{\prime \prime}\right),
\end{aligned}
$$

because

$$
\mathrm{G}_{i}-\mathrm{G}_{i}^{\prime} \cdot \overline{\mathbb{F}}_{k}^{\prime-1} \cdot \overline{\mathbb{F}}_{k}=\left[\mathrm{G}_{i}^{\prime}-\mathrm{G}_{i}^{\prime} \cdot \overline{\mathrm{F}}_{k}^{\prime-1} \overline{\mathbb{F}}_{k}^{\prime}, \overline{\mathrm{G}}_{i}^{\prime \prime}-\mathrm{G}_{i}^{\prime} \cdot \overline{\mathbb{F}}_{k}^{\prime-1} \cdot \overline{\mathrm{F}}_{k}^{\prime \prime}\right]=\left[0, \overline{\mathrm{G}}_{i}^{\prime \prime}-\mathrm{G}_{i}^{\prime} \cdot \overline{\mathbb{F}}_{k}^{\prime-1} \cdot \overline{\mathbb{F}}_{k}^{\prime \prime}\right]
$$

Decomposing the equality (3.7) into two equalities like (3.3) and (3.4), we obtain

$$
\begin{aligned}
& \frac{\partial}{\partial x_{j}}\left(\tilde{\mathbf{G}}_{i}^{\prime \prime}-\tilde{\mathbf{G}}_{i}^{\prime} \cdot \overline{\mathbb{F}}_{k}^{\prime-1} \cdot \overline{\mathbb{F}}_{k}^{\prime \prime}\right)-\left(\tilde{\mathbf{G}}_{i}^{\prime \prime}-\tilde{\mathbf{G}}_{i}^{\prime} \cdot \overline{\mathbb{F}}_{k}^{\prime-1} \cdot \overline{\mathbb{F}}_{k}^{\prime \prime}\right) \cdot\left(\tilde{\mathbf{G}}_{j}^{\prime \prime}-\tilde{\mathbf{G}}_{j}^{\prime} \cdot \overline{\mathbb{F}}_{k}^{\prime-1} \cdot \overline{\mathbb{F}}_{k}^{\prime \prime}\right) \\
= & \frac{\partial}{\partial x_{i}}\left(\tilde{\mathbf{G}}_{j}^{\prime \prime}-\tilde{\mathbf{G}}_{j}^{\prime} \cdot \overline{\mathbb{F}}_{k}^{\prime-1} \cdot \overline{\mathbb{F}}_{k}^{\prime \prime}\right)-\left(\tilde{\mathbf{G}}_{j}^{\prime \prime}-\tilde{\mathbf{G}}_{j}^{\prime} \cdot \overline{\mathbb{F}}_{k}^{\prime-1} \cdot \overline{\mathbb{F}}_{k}^{\prime \prime}\right) \cdot\left(\tilde{\mathbb{G}}_{i}^{\prime \prime}-\tilde{\mathbb{G}}_{i}^{\prime} \cdot \overline{\mathbb{F}}_{k}^{\prime-1} \cdot \overline{\mathbb{F}}_{k}^{\prime \prime}\right)
\end{aligned}
$$

i.e. the compatibility conditions for the system (3.5) are identically satisfied.

Next we discuss the usefulness of the previous result. Indeed, if a linear system (3.1) with some linear homogeneous algebraic constraints is given, then using the algorithm from [6] we reduce it to a system of dimension less than the former one without any constraints and the compatibility conditions are identically satisfied. Then using Theorem 2.1, we can expand the solution as a convergent functional series. Specially, let us consider the system (3.1) and let the compatibility conditions be not identically satisfied for all initial conditions. In [8] the necessary and sufficient conditions are given for the existence of a solution for given initial conditions. These conditions are algebraic constraints. Now applying the algorithm in [6], we reduce the given system to a system of dimension less than the former one and the compatibility conditions are identically satisfied for all initial conditions. Then Theorem 2.1 can be applied to obtain the required solution.

Remark. Finally note that if we consider a system of nonlinear equations instead of (3.1), then a theorem like Theorem 3.1 holds for that system because as it is shown in [7], the nonlinear systems can be considered as linear systems of infinitely many equations and infinitely many unknown functions $y_{\alpha}$. The above discussion considering the initial conditions is still valid for nonlinear systems of PDEs.

\section{Example}

Let the following system of four PDEs be given

$$
\frac{\partial y_{1}}{\partial x_{1}}-\alpha \cdot\left(y_{1}+y_{2}\right)=0
$$




$$
\begin{array}{r}
\frac{\partial y_{2}}{\partial x_{1}}+\left(x_{1}-x_{3}\right) \cdot y_{1}-\left(x_{1}+x_{2}\right) \cdot y_{2}=0 \\
\frac{\partial y_{1}}{\partial x_{2}}-\beta \cdot\left(y_{1}-y_{2}\right)=0 \\
x_{2} \cdot \frac{\partial y_{2}}{\partial x_{2}}-\beta \cdot\left(x_{3} \cdot y_{1}+x_{1} \cdot y_{2}\right)=0
\end{array}
$$

where $\alpha=\frac{1}{x_{1}+2 x_{2}-x_{3}}, \beta=\frac{1}{x_{1}+x_{3}}$. If we define

$$
\mathbf{y}=\left[\begin{array}{l}
y_{1} \\
y_{2}
\end{array}\right] \quad \text { and } \quad \mathbf{x}=\left[\begin{array}{l}
x_{1} \\
x_{2} \\
x_{3}
\end{array}\right]
$$

then we bring the system (4.1)-(4.4) into the form (3.1), where $p=2$ and

$$
\mathrm{G}_{1}(x)=\left[\begin{array}{cc}
-\alpha & -\alpha \\
x_{2}-x_{3} & -x_{1}-x_{2}
\end{array}\right], \quad \mathrm{G}_{2}(x)=\beta \cdot\left[\begin{array}{cc}
-1 & 1 \\
-\frac{x_{3}}{x_{2}} & -\frac{x_{1}}{x_{2}}
\end{array}\right] .
$$

Hence we obtain

$$
\begin{aligned}
\mathrm{G}_{1} \cdot \mathrm{G}_{2}-\mathrm{G}_{2} \cdot \mathrm{G}_{1} & =\beta \cdot\left[\begin{array}{cc}
\alpha \cdot \frac{x_{3}}{x_{2}}-x_{2}+x_{3} & -2 \cdot \alpha+\alpha \cdot \frac{x_{1}}{x_{2}}+x_{1}+x_{2} \\
-\alpha \cdot \frac{x_{3}}{x_{2}}+x_{1}-x_{2}+2 \cdot x_{3} & -\alpha \cdot \frac{x_{3}}{x_{2}}+x_{2}-x_{3}
\end{array}\right], \\
\frac{\partial \mathrm{G}_{1}}{\partial x_{2}}-\frac{\partial \mathrm{G}_{2}}{\partial x_{1}} & =\left[\begin{array}{cc}
2 \cdot \alpha^{2}-\beta^{2} & 2 \cdot \alpha^{2}+\beta^{2} \\
1-\beta^{2} \cdot \frac{x_{3}}{x_{2}} & -1-\beta^{2} \cdot \frac{x_{1}}{x_{2}}+\frac{\beta}{x_{2}}
\end{array}\right] .
\end{aligned}
$$

Thus

$$
\begin{aligned}
\mathbb{F}_{1}=\frac{\partial \mathbb{G}_{1}}{\partial x_{2}}-\frac{\partial \mathbf{G}_{2}}{\partial x_{1}}-\left(\mathbb{G}_{1} \cdot \mathbf{G}_{2}-\mathbf{G}_{2} \cdot \mathbb{G}_{1}\right) \\
=\left[\begin{array}{cc}
2 \cdot \alpha^{2}-\beta^{2}-\beta \cdot\left(\alpha \cdot \frac{x_{3}}{x_{2}}-x_{2}+x_{3}\right) & 2 \cdot \alpha^{2}+\beta^{2}-\beta \cdot\left(-2 \cdot \alpha+\alpha \cdot \frac{x_{1}}{x_{2}}+x_{1}+x_{2}\right) \\
1-\beta^{2} \cdot \frac{x_{3}}{x_{2}}-\beta \cdot\left(-\alpha \cdot \frac{x_{3}}{x_{2}}+x_{1}-x_{2}+2 \cdot x_{3}\right) & -1-\beta^{2} \cdot \frac{x_{1}}{x_{2}}+\frac{\beta}{x_{2}}-\beta \cdot\left(-\alpha \cdot \frac{x_{3}}{x_{2}}+x_{2}-x_{3}\right)
\end{array}\right] .
\end{aligned}
$$

It can be verified that $\operatorname{det} \mathbb{F}_{1}=0$, thus $\mathbb{F}_{1}=\left[\begin{array}{l}\overline{\mathbb{F}}_{1} \\ \tilde{\mathbb{F}}_{1}\end{array}\right]$, where $\overline{\mathbb{F}}_{1}$ and $\tilde{\mathbb{F}}_{1}$ are (linearly dependent) vector-rows. It can be verified also that, by further differentiation, new linearly independent rows cannot be obtained, thus $k=1$. Following the theory in Section 3, we partition the vector function $\mathbf{y}$ into $\mathbf{y}=\left[\begin{array}{l}y_{1} \\ y_{2}\end{array}\right]=\left[\begin{array}{l}y^{\prime} \\ y^{\prime \prime}\end{array}\right]$. It can be verified that the equation

$$
\overline{\mathbf{F}}_{1} \cdot \mathbf{y}=0
$$

is equivalent to

$$
y^{\prime}=\frac{x_{1}+x_{2}}{x_{2}-x_{3}} \cdot y^{\prime \prime}
$$


The system (4.1)-(4.4) becomes

$$
\begin{aligned}
\frac{\partial y^{\prime}}{\partial x_{1}}-\alpha \cdot\left(y^{\prime}+y^{\prime \prime}\right) & =0 \\
\frac{\partial y^{\prime \prime}}{\partial x_{1}}+\left(x_{1}-x_{3}\right) \cdot y^{\prime}-\left(x_{1}+x_{2}\right) \cdot y^{\prime \prime} & =0 \\
\frac{\partial y^{\prime}}{\partial x_{2}}-\beta \cdot\left(y^{\prime}-y^{\prime \prime}\right) & =0 \\
\frac{\partial y^{\prime \prime}}{\partial x_{2}}-\beta \cdot\left(\frac{x_{3}}{x_{2}} \cdot y^{\prime}+\frac{x_{1}}{x_{2}} \cdot y^{\prime \prime}\right) & =0 .
\end{aligned}
$$

According to the theory in Section 3, the equations (4.6) and (4.8) are consequences of the equations (4.7) and (4.9), respectively. Thus the original system of PDEs (4.1)-(4.4) is reduced to (3.5), i.e.

$$
\begin{aligned}
& \frac{\partial y^{\prime \prime}}{\partial x_{1}}=0 \\
& \frac{\partial y^{\prime \prime}}{\partial x_{2}}-\frac{1}{x_{1}-x_{3}} \cdot y^{\prime \prime}=0 .
\end{aligned}
$$

According to Theorem 3.1, the compatibility conditions for this system are identically satisfied. Thus, we have reduced the original system of four PDEs with two unknown functions not satisfying the compatibility conditions identically to the system of two PDEs with one unknown function satisfying the compatibility conditions identically. For obtaining the solution, we can apply the theory of Section 2, i.e. the functional expansion method presented by Theorem 2.1. Suppose the following initial conditions

$$
y_{1}\left(0,0, x_{3}\right)=0, \quad y_{2}\left(0,0, x_{3}\right)=\varphi\left(x_{3}\right)
$$

are given, where $\varphi$ is a function. Note that the initial conditions have to satisfy the equation (4.5) for $x_{1}=x_{2}=0$, i.e. $\overline{\mathrm{F}}_{1}\left(0,0, x_{3}\right) \cdot \mathrm{y}\left(0,0, x_{3}\right)=0$, and they are chosen to satisfy it. The initial condition for the reduced system (4.10)-(4.11) is $y^{\prime \prime}\left(0,0, x_{3}\right)=$ $\varphi\left(x_{3}\right)$.

We have to find (scalar) functions $P^{<w_{1}, w_{2}>}\left(x_{1}, x_{2}, x_{3}\right)$ satisfying

$$
\begin{aligned}
P^{<0,0>} & =0, \\
P^{\left.<w_{1}+1, w_{2}\right\rangle} & =\frac{\partial}{\partial x_{1}} P^{\left.<w_{1}, w_{2}\right\rangle}, \\
P^{\left.<w_{1}, w_{2}+1\right\rangle} & =\frac{\partial}{\partial x_{2}} P^{\left\langle w_{1}, w_{2}\right\rangle}-\frac{1}{x_{2}-x_{3}} \cdot P^{\left.<w_{1}, w_{2}\right\rangle} .
\end{aligned}
$$

The functions $P^{<w_{1}, w_{2}>}=0$ for $w_{1} \neq 0$ and

$$
P^{<0, w_{2}>}=(-1)^{w_{2}} \cdot \frac{w_{2} !}{\left(x_{2}-x_{3}\right)^{w_{2}}} .
$$


According to (2.5) the functional expansion for $y^{\prime \prime}$ is given by

$$
\begin{aligned}
y^{\prime \prime} & =\left(\sum_{w_{1}=0}^{\infty} \sum_{w_{2}=0}^{\infty} \frac{\left(-x_{1}\right)^{w_{1}}}{w_{1} !} \cdot \frac{\left(-x_{2}\right)^{w_{2}}}{w_{2} !} \cdot P^{<w_{1}, w_{2}>}\right) \varphi\left(x_{3}\right) \\
& =\left(\sum_{w_{2}=0}^{\infty} \frac{\left(-x_{2}\right)^{w_{2}}}{w_{2} !} \cdot P^{<0, w_{2}>}\right) \cdot \varphi\left(x_{3}\right)=\varphi\left(x_{3}\right) \cdot \sum_{w_{2}=0}^{\infty}\left(\frac{x_{2}}{x_{2}-x_{3}}\right)^{w_{2}} .
\end{aligned}
$$

For $\left|\frac{x_{2}}{x_{2}-x_{3}}\right|<1$ this series converges to $y^{\prime \prime}=-\frac{\left(x_{2}-x_{3}\right)}{x_{3}} \cdot \varphi\left(x_{3}\right)$ which means that the solution of the system (4.1)-(4.4) with Cauchy initial conditions (4.12) is given by

$$
y_{1}=-\frac{\left(x_{1}+x_{2}\right)}{x_{3}} \cdot \varphi\left(x_{3}\right), \quad y_{2}=-\frac{\left(x_{2}-x_{3}\right)}{x_{3}} \cdot \varphi\left(x_{3}\right)
$$

The present example was included following the referee's suggestion.

\section{References}

[1] E. Goursat, Leçons sur le Problème de Pfaff, Librairie Scientifique J. Hermann, Paris, 1922.

[2] C. Chevalley, Theory of Lie Groúps, Princeton University Press, Princeton, 1946.

[3] P. K. Raševskii, Geometrical Theory of Equations with Partial Derivatives, Gostehizdat, Moscow-Leningrad, 1947 (in Russian).

[4] N. Saltikov, Methods for Integration of Partial Differential Equations of the First Order of One Unknown Function, Serbian Academy of Sciences, Beorgrad, 1947 (in Serbo-Croatian).

[5] J. Stefanovski and G. Dimirovski, Prilozi kon diferencijalno-geometriskiot priod za rešavanje na rospredeleno-parametarski dinamički sistemi, $V$ Symposium of Differential Equations, Association of Mathematicians and Informaticians of Macedonia, Ohrid, 1995.

[6] J. Stefanovski, K. Trenčevski and V. Covachev, Charpit system of partial equations with algebraic constraints, submitted for publication.

[7] K. Trenčevski, Solution of analytical system of partial differential equations, Serdica Math. J. 21(1995), 171-184.

[8] $\mathrm{K}$. Trenčevski, On the parallel vector fields in vector bundles, submitted for publication in Tensor.

JVP HS Streževo, bul. 1 Maj b. b., 97000 Bitola, Macedonia.

E-mail: strezevo@lotus.mpt.com.mk

Institute of Mathematics, St. Cyril and Methodius University, P. O. Box 162, 91000 Skopje, Macedonia.

E-mail: kostatre@iunona.pmf.ukim.edu.mk

Institute of Mathematics, Bulgarian Academy of Sciences, 8 Acad. G. Bonchev Str., 1113 Sofia, Bulgaria.

E-mail: matph@math.bas.bg 